University of Massachusetts Amherst

ScholarWorks@UMass Amherst

Chemistry Department Faculty Publication Series

Chemistry

1999

\title{
High-performance, flow-based, sample pre- treatment and introduction procedures for analytical atomic spectrometry
}

JF Tyson

Follow this and additional works at: https://scholarworks.umass.edu/chem_faculty_pubs

Part of the Chemistry Commons

\section{Recommended Citation}

Tyson, JF, "High-performance, flow-based, sample pre-treatment and introduction procedures for analytical atomic spectrometry" (1999). Journal of Analytical Atomic Spectrometry. 1056.

Retrieved from https://scholarworks.umass.edu/chem_faculty_pubs/1056 


\title{
High-performance, flow-based, sample pre-treatment and introduction procedures for analytical atomic spectrometry $\dagger$
}

\author{
Plenary Lecture
}

\author{
Julian F. Tyson \\ Department of Chemistry, University of Massachusetts, Box 34510 Amherst, MA 01003-4510, USA
}

Received 8th September 1998, Accepted 8th December 1998

Recent and on-going work in the author's laboratory is described with particular reference to the use of flow injection, continuous flow and HPLC procedures for the development of improved analytical methodology for (a) the determination of trace concentrations of $\mathrm{As}, \mathrm{Cd}, \mathrm{Pb}$ and $\mathrm{Se}$ and $(\mathrm{b})$ the determination of various arsenic and selenium compounds. The methods have been applied to the analysis of several different sample matrices, including urine, soil, sediments, waters, plants (garlic, onion, apple leaves), yeast, fruit juices, wine, calcium supplements and marine plankton. The dependence of the LOD of an FI HG method on sample volume is examined and the validity of the proposed rectangular hyperbolic relationship established for a number of different analyses. The use of immobilized tetrahydroborate in conjunction with preconcentration of the analyte on the same anion-exchange resin is described as a possible procedure for improving the LOD. When used in conjunction with ETAAS, an LOD of $0.004 \mu \mathrm{g}^{-1}$ for both As and Se was obtained for a sample volume of $10 \mathrm{ml}$. The procedure was also used in a method for the determination of inorganic arsensic and methylated arsenic(v) species. Methods for the determination of $\mathrm{Pb}$ by $\mathrm{HG}$ in the presence of hexacyanoferrate(III) were developed and applied to the analysis of urine, soils, waters and apple leaves. In the case of urine, the interference from the chelating agents used in the treatment of patients with elevated lead was overcome by the addition of Sc. The best LOD of $0.03 \mu \mathrm{g} 1^{-1}$ was obtained for a procedure in which the lead hydride was trapped on the interior of a flame-heated slotted quartz tube under fuel-lean conditions with subsequent atomization when the flame was made fuel rich (by the injection of a small volume of isobutyl methyl ketone). It has been confirmed that it is possible to determine Cd by a 'cold vapour' procedure and it was shown that the nature of the atom cell surface played no part in the atomization process, which appeared to be the spontaneous decomposition of the species evolved from acid solution of cadmium on the addition of sodium tetrahydroborate solution. A modest increase in signal was obtained in the presence of nickel and thiourea. An LOD of $0.02 \mu \mathrm{g}^{-1}$ was obtained for a sample volume of $300 \mu \mathrm{l}$ and the procedure was used for the analysis of NIST SRM 2711 (Montana Soil) in which the interference from the high lead content was overcome by coprecipitation with barium sulfate. Three examples of procedures using manifold designs incorporating an 'eight-port' rotary valve are given to illustrate the versatility of this component: the separation of high concentrations of uranium $\left(5000 \mathrm{mg}^{-1}\right)$ from trace concentrations $\left(1 \mu \mathrm{g} \mathrm{1}^{-1}\right)$ of $\mathrm{Al}, \mathrm{Be}, \mathrm{Li}$ and $\mathrm{Mg}$ for determination by ICP-MS, the automated implementation of the co-immobilization of analyte and tetrahydroborate on an anion-exchange resin and the stopped-flow microwave digestion of human urine for the determination of Se by HG and ETAAS. Improvements in the ion-pair (with trichloroacetate) reversed-phase $\left(\mathrm{C}_{8}\right)$ HPLC procedure (with ICP-MS detection) for the separation of selenoamino acids (and closely related compounds) were made with a new stationary phase (Waters Symmetryshield $\mathrm{RP}_{8}$ ) and a small-volume spray chamber. The results of extraction procedures indicated that much of the selenium in yeast and garlic is bound in high molecular mass material. So far only a few of the compounds in the extracts have been identified by retention time matching. A reversed phase $\left(\mathrm{C}_{18}\right)$, ion-pair (tetrabutylammonium) HPLC procedure for the separation of four arsenic species (arsenite, arsenate, monomethylarsinate and dimethylarsonate), with detection by post-column HG-AAS, has been devised and applied to the extracts of soils spiked with these four species. Low recoveries of arsenite were obtained and microwave energy significantly accelerated the oxidation of arsenite to arsenate.

There are many areas of scientific study in which information about the chemical composition, in terms of the trace or minor elemental composition of the relevant materials, is needed as part of the problem-solving strategy. A good example of such an area is the study of the biogeochemical cycling of the elements. For some elements, only the most rudimentary knowledge has so far been accumulated, and for others, the relative magnitude of the contributing processes are unknown. There are many subsidiary problems to be tackled, such as the elucidation of the chemical basis of the cancer-suppressing

$\dagger$ Presented at the Ninth Biennial National Atomic Spectroscopy Symposium (BNASS), Bath, UK, July 8-10, 1998. and cancer-promoting properties of some elements and their relevant compounds.

In a recent overview of the future of atomic spectrometry for environmental analysis, Sturgeon ${ }^{1}$ identified a number of trends and driving forces which probably have general applicability in the wider context of trace element determinations, namely: (a) more elements will be sought at lower concentrations, (b) total element determinations are becoming less relevant and speciation is becoming more relevant, (c) there is a greater need to minimize contamination as a consequence of enhanced instrumental LODs, and (d) there is a need for reduced waste production and reagent consumption.

Sturgeon also pointed out that 'Real sample matrices may 
give rise to a plethora of potential problems, often associated with high dissolved solids content, including suppression of sensitivity, increased background levels and spectral interferences. The practical consequence is that, if the effects cannot be remedied by simple dilution, matrix separation and concentration of the analyte(s) becomes necessary. This is often the case in any event, as many samples from uncontaminated sources contain analytes of interest at concentration levels which challenge the best instrumental limits of detection'. With regard to the future, he suggested that 'Automation of many routine sample preparation tasks can be anticipated. Enhanced LODs can be achieved with direct solids (e.g., LA) and slurry sampling approaches as well as through more extensive use of on-line chemical manifolds and FI techniques to minimize sample contamination and perform matrix separation-analyte concentration functions.... Without doubt, the greatest impact on sample processing and introduction for atomic spectrometry has derived from the fields of FI technology and microwave radiation'.

Finally, Sturgeon concluded, 'Current instrumentation is already capable of providing instrumental detection limits far superior to method detection limits for many elements due to our inability to control contamination and, hence, the method blank. It is to be hoped that the widespread use of FI techniques and, ultimately, perhaps nanotechnology for sample handling will help alleviate this particular problem'.

However, if one takes a broad view of the current research and development efforts into improving analytical methodologies in which commercially available atomic spectrometric instrumentation is used, then it is apparent that the commonly used techniques have limitations which, in turn, set performance limits to the methods in which they are incorporated. Thus, FAAS does not have LODs low enough, it has a limited working range and limited element coverage and suffers from matrix interferences; ETAAS suffers from the last three of these limitations, but has instrumental LODs which are so low that method LODs are governed by blank contamination; ICP-OES has a wider elemental coverage (and offers the possibility of genuine simultaneous multi-element determinations) and a larger working range but instrumental LODs not good enough for many environmental and clinical applications, and suffers from spectral interferences; and ICP-MS has low instrumental LODs (blank contamination becomes a problem again), with wide elemental coverage and nearly simultaneous multi-element determinations but the instrumentation is delicate and suffers from various matrix interference effects. It is clear from the review literature covering these techniques (as exemplified by the Atomic Spectrometry Updates which appear in the June, August and October issues of this journal) that much of the published work relating to the use of these techniques concerns some aspect of the limitations listed above. For some techniques, e.g., ETAAS, the majority of technique-based publications are concerned with achieving a separation between analyte and matrix: either by the judicious choice of some thermochemical reactions within the atomizer, or by the use of powerful background correction procedures, or both.

In essence, the contribution of FI techniques to this area of analytical instrumental analysis has been to open up a number of possibilities for the separation of analyte and matrix with analyte concentration. As these two functions are almost always achieved by appropriate chemical reactions, the use of FI techniques in this fashion has been described ${ }^{2}$ as 'putting the chemistry back into analytical chemistry'.

\section{Flow injection, analyte concentration and detection limits}

Numerous publications appear each year describing procedures in which the LOD has been improved over that which would be obtained by direct introduction of the sample (as received or as produced by some dissolution and/or digestion procedure). This is particularly so for methods in which the authors wish to use FAAS as the instrumental technique. ${ }^{3}$ This goal is, in itself, reasonable; FAAS is, after all, a well understood technique, is robust and relatively inexpensive, and requires a minimum of operator skill. By far the most popular procedure for analyte concentration and matrix separation is solid phase extraction (SPE). In general, the analyte from a relatively large volume of solution is selectively retained by a solid reagent phase (by a variety of mechanisms) and then released into a relatively small volume of eluent.

In addition, chemical vapour generation $(\mathrm{CVG})$, i.e., $\mathrm{HG}$ and $\mathrm{CV}$ procedures, are the procedures of choice ${ }^{4}$ for the determination of As, Se and $\mathrm{Hg}$, and probably also for the determination of $\mathrm{Sb}, \mathrm{Bi}$ and $\mathrm{Ge}$. In general, the analyte is selectively converted into a volatile derivative which is then blown out of solution. Both of these procedures give rise to an increased signal owing to the greater analyte mass flux introduced to the atomizer than would be the case for the conventional nebulization of the original sample solution. When used for ETAAS (with in-atomizer trapping in the case of $\mathrm{CVG}$ ), the procedures have the effect of introducing a greater mass of analyte into the atomizer than that which would have been introduced in a conventional sample volume (say $20 \mu \mathrm{l}$ ). Again, an increased signal is obtained. It is therefore tempting to argue, as many workers do, that this increased sensitivity gives rise to increased detection limits and that there is a relationship between LOD and sample volume such that the LOD can be decreased to any desired value; it is simply a matter of preconcentrating a sufficiently large sample volume.

\section{Detection limits and sample volume}

A recent survey ${ }^{5}$ (by no means exhaustive) of relevant papers in the area of HG-ETAAS found as many as 15 papers in which this kind of claim was made. This is not surprising as, in general, it is true that LODs get better as the sample volume is increased. However, it is decidedly misleading to give the impression that there is no limit to how low the LOD can be. The relationship between these two quantities needs to be examined a little more closely and, in particular, the situation that pertains when an FI procedure is used.

In a recent paper on the determination of $\mathrm{Cd}$ by CVGETAAS with in-atomizer trapping, Geonaga Infante et al. provided $^{6}$ the following information: "the detection limit was calculated to be $60 \mathrm{ng} \mathrm{l}^{-1}$ for $1.4 \mathrm{ml}$... of course, lower detection limits could be obtained if higher sample volumes were preconcentrated...7 ml...resulted in a detection limit of $13 \mathrm{ng} 1^{-1}$. A scatter plot (see Fig. 1) of these data with LOD (on the ordinate) against sample volume, $V$, (on the abscissa) would have two points. The relationship between LOD, $C_{\mathrm{L}}$ $\left(\mu \mathrm{g}^{-1}\right)$, and $V(\mathrm{ml})$ could be the straight line, $C_{\mathrm{L}}=-8.39 \times$ $10^{-3} V+0.0718$. However, this would predict that the LOD was zero for a sample volume of about $8.6 \mathrm{ml}$ and that for sample volumes greater than this, the LOD would be negative! A more thoughtful analysis of the situation would lead to the conclusion that the relationship would be more properly described by one of inverse proportion, i.e., as the signal (for a given concentration) is directly proportional to sample volume, it might be expected that the LOD would be inversely proportional to $V$. For the data in Fig. 1, the relationship would be $C_{\mathrm{L}}=b / V$, where $b$ is a parameter to be fitted. The data, in fact, do not fit this function particularly well as the two values calculated for $b$ are $8.4 \times 10^{-3}$ and $9.1 \times 10^{-3} \mu \mathrm{g} \mathrm{ml}^{2}$. Another iteration of the modelling process is needed.

Detection limit is a function of signal-to-noise ratio and 


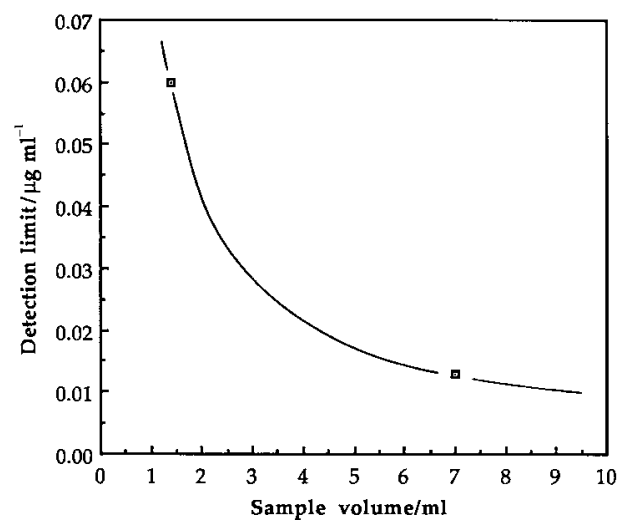

Fig. 1 Relationship between limit of detection and sample volume for HG-ETAAS determination of cadmium with in-atomizer trapping. Data are taken from ref. 6 . The curve is a rectangular hyperbola of the form $y=b / x+a$, where $a$ and $b$ are constants. Details of the equation are given in the text

may be defined ${ }^{7}$ as the concentration giving a signal equal to three times the standard deviation of the blank signal. If the slope of the calibration is $S$ (in appropriate units) and standard deviation of blank signal is $s_{\mathrm{bl}}$, then the LOD is given by $C_{\mathrm{L}}=$ $3 s_{\mathrm{bl}} / S$. In general, it might be expected that both $S$ and $s_{\mathrm{bl}}$ would be functions of $V$, with $S$ directly proportional to $V$ for those situations in which the instrument response is directly proportional to analyte mass. In general, $s_{\mathrm{b} 1}$ will be made up of a contribution from the instrument, $s_{0}$, and a contribution due to the response to the reagents (including the analyte contamination in the reagents), $s_{\mathrm{R}}$.

For a batch procedure, the amounts of reagents are independent of sample volume, hence the LOD- $V$ relationship is $C_{\mathrm{L}}=3 s_{\mathrm{b} 1} / k V$, where $k$ is the constant of proportionality between sensitivity and sample volume.

The detection limit can be as low as one wishes, simply by increasing the sample volume, but there is a practical limit set by the amount of sample needed and the time taken to process this sample volume. For example, in the determination of $\mathrm{Cd}$ cited above, if this model were valid it would require a sample volume of about $88 \mathrm{ml}$ to obtain an LOD of $0.001 \mu \mathrm{g} 1^{-1}$; at a flow rate of $2.8 \mathrm{ml} \mathrm{min}^{-1}$, it would take about $30 \mathrm{~min}$ to process just one sample. As sample processing times become this long, instrumental drift becomes a contributor to the noise on the signal.

In a flow-based procedure, whether it involves HG or SPE, the amount of reagent used is directly related to sample volume and thus the contribution to $s_{\mathrm{bl}}$ from $s_{\mathrm{R}}$ increases in direct proportion to the sample volume so that $s_{\mathrm{bl}}=\mathrm{s}_{0}+k V$ (where $k$ is the constant of proportionality relating $s_{\mathrm{R}}$ and $V$ ) and $C_{\mathrm{L}}=3 s_{\mathrm{bl}} / k V=3\left(s_{0}+k V\right) / k V=3 s_{0} / k V+3 k / k$. This equation has the general form $C_{\mathrm{L}}=b / V+a$, and will be referred to as the flow injection detection limit (or FIDL) equation. For the data used as example, the parameters $a$ and $b$ may be calculated (as two points are available) to give an FIDL equation $C_{\mathrm{L}}=$ $8.23 \times 10^{-3} / V+0.00125$. This equation is also shown in Fig. 1 .

\section{Detection limits, sample volume and reagent blank}

A more detailed treatment of the problem, which attempted to identify the factors contributing to the $a$ and $b$ terms, has been presented, ${ }^{5}$ together with results for the determination of As by FI-HG-ETAAS for 10 sample volumes covering the range $0.156-1.56 \mathrm{ml}$. The data were consistent with an FIDL equation $C_{\mathrm{L}}=0.041 / V+0.007$, from which it was concluded, with a certain degree of subjectivity based on estimations of the confidence intervals about the LODs, that no significant improvement in LOD would be obtained for a sample volumes in excess of $1.00 \mathrm{ml}$ ( $\mathrm{LOD} 0.05 \mu \mathrm{g}^{-1}$ ). The model indicated that the major contribution to the $a$ term was the concentration of analyte in the reagents. Results supporting this finding had already been obtained ${ }^{8}$ for the determination of $\mathrm{Se}$ in urine by FI-HG-ETAAS. It was found (a) that the major source of analyte contamination was the borohydride reagent and (b) the best LOD $\left(0.06 \mu \mathrm{g}^{-1}\right.$ in the solution introduced into the manifold) was obtained for an injection volume of $1 \mathrm{ml}$. However, more data are needed before it may be concluded that for all FI-HG-ETAAS procedures the optimum sample volume is $1 \mathrm{ml}$.

\section{Immobilized tetrahydroborate reagent}

It may be concluded that a significant improvement in LOD would be obtained for an FI procedure if the reagent volume were independent of sample volume. One possibility for $\mathrm{HG}$ procedures would be to use an immobilized tetrahydroborate reagent. Tesfalidet and Irgum described such a procedure for the determination of $\mathrm{As}^{9}$ and $\mathrm{Se}^{10}$ in which the tetrahydroborate was immobilized on an anion-exchange resin and the hydride was generated on passage of an acidified sample solution. It was observed ${ }^{11}$ that the analyte could also be retained on the resin and, as the first stage in developing methods based on immobilized tetrahydroborate reagent, the performance of a procedure in which analyte (in this case selenite) and the borohydride were co-immobilized was evaluated. It was found ${ }^{11}$ that (a) the optimum tetrahydroborate concentration was $0.05 \%$, considerably lower than values typically used in conventional flow or batch procedures, (b) the procedure gave signals for both $\mathrm{As} s^{\mathrm{III}}$ and $\mathrm{As}^{\mathrm{V}}$ but only for $\mathrm{Se}^{\mathrm{IV}}$ (not for $\mathrm{Se}^{\mathrm{VI}}$ ) and (c) the LOD decreased as the sample volume increased. The procedure was applied to the determination of Se in river, lake and tap water; no interferences from these matrices were encountered for Se concentrations between 0.5 and $10 \mu \mathrm{g}^{-1}$. Detection limits were measured for three sample volumes, 3,6 and $9 \mathrm{ml}$, for which LODs of $0.24,0.15$ and $0.12 \mu \mathrm{g}^{-1}$, respectively, were obtained. These results are consistent with the FIDL equation $C_{\mathrm{L}}=0.540 / V+0.060$ (the correlation coefficient of a least-squares fit of a straight line to points on a plot of $C_{\mathrm{L}}$ versus $1 / V$ is 1.0000$)$.

\section{Analyte concentration by SPE}

The next stages in the development of this procedure were (a) to separate the retention of the tetrahydroborate and the analyte, so that the amount of reagent used was independent of the volume of sample loaded, (b) trap the generated hydride on the interior of a graphite furnace and (c) automate the procedure with a commercially available computer-controlled flow injection analyzer unit [a Perkin-Elmer (Norwalk, CT, USA) FIAS 200]. The separate sample loading step meant that the sample volume could be increased substantially without increasing the blank contribution and it was found ${ }^{12}$ that for a $20 \mathrm{ml}$ sample volume (loaded at $20 \mathrm{ml} \mathrm{min}^{-1}$ ), the LOD was $0.004 \mu \mathrm{g} \mathrm{l}^{-1}$. The amount of tetrahydroborate used per determination was $5 \mathrm{mg}$ ( $5 \mathrm{ml}$ of $0.1 \% \mathrm{~m} / \mathrm{v}$ solution flowing at $5.0 \mathrm{ml} \mathrm{min}{ }^{-1}$ for $\left.1.0 \mathrm{~min}\right)$. The LOD was significantly improved over that which would have been obtained for a sample volume of $20 \mathrm{ml}$ with the quartz tube atomizer and the simultaneous immobilization procedure. Substitution in the above FIDL equation gives a value of $0.087 \mu \mathrm{g} \mathrm{ml}^{-1}$. The procedure was applied ${ }^{13}$ to the determination of Se in several fresh garlic samples. Values ranging from 55 to $350 \mu \mathrm{g} \mathrm{kg}^{-1}$ were found.

\section{Application to speciation of arsenic compounds}

As the arsenic species $\mathrm{AsO}(\mathrm{OH})_{3}, \mathrm{As}(\mathrm{OH})_{3}, \mathrm{CH}_{3} \mathrm{AsO}(\mathrm{OH})_{2}$ (MMA) and $\left(\mathrm{CH}_{3}\right)_{2} \mathrm{AsO}(\mathrm{OH})$ (DMA) are weak acids with sufficiently different $\mathrm{p} K_{\mathrm{a}}$ values, a procedure based on selective 


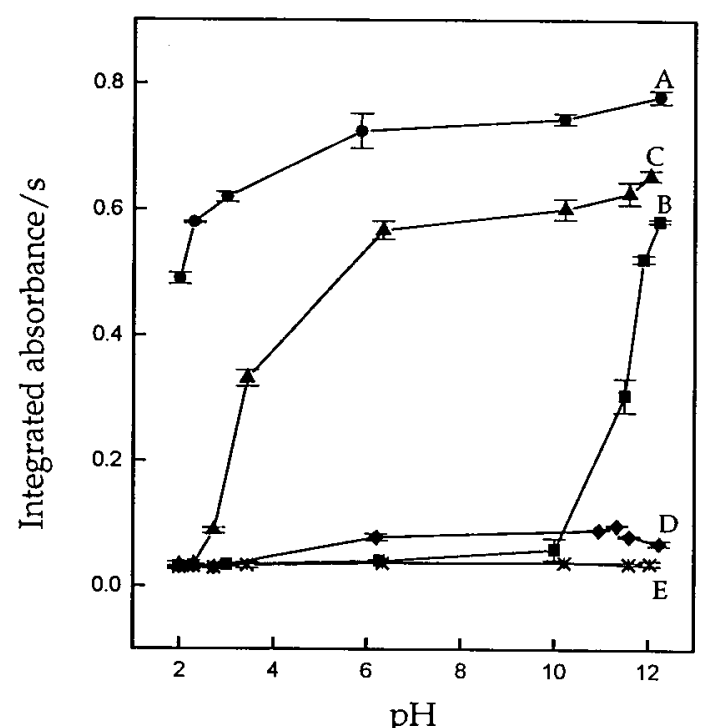

Fig. 2 Effect of sample $\mathrm{pH}$ on peak are a signal for four arsenic species and for the blank: A, arsenate; $\mathrm{B}$, arsenite; $\mathrm{C}$, monmethylarsinate; D, dimethylarsonate; and E, blank. The error bars are the standard deviations of five replicate measurements.

analyte retention on the anion-exchange resin is possible. The $\mathrm{p} K_{\mathrm{a}}$ values are $\mathrm{As}^{\mathrm{v}}, 2.3, \mathrm{As}^{\mathrm{III}}, 9.2$, monomethylarsenic $(\mathrm{v}), 4.0$ and dimethylarsenic(v) 6.3, hence at low $\mathrm{pH}$ values $\mathrm{As}^{\mathrm{v}}$ will exist as an anion whereas all the other species will be fully protonated and not retained. The results of the $\mathrm{pH}$ study are shown in Fig. 2, confirming that this predicted behaviour is indeed obtained. A speciation procedure was devised ${ }^{14}$ based on selective retention of $\mathrm{As}^{\mathrm{v}}$ (at $\mathrm{pH} 2.3$ ) and selective oxidation of the other species to $\mathrm{As}^{\mathrm{V}}$. Three stages were needed: in the first, only $\mathrm{As}^{\mathrm{V}}$ was determined; in the second, $\mathrm{As}^{\mathrm{III}}$ was selectively oxidized with hydrogen peroxide and nitric acid so the difference between the result for this step and that for the first step gave the As ${ }^{\text {III }}$ content; in the third stage, all species were oxidized to $\mathrm{As}^{\mathrm{V}}$ by alkaline peroxodisulfate in a sealed vessel in a microwave field, so the difference between the result for this step and that for the second step gave the concentration of the methylated arsenic species.

A fully automated system was constructed around a PerkinElmer FIAS 200 unit interfaced to an ETA atomic absorption spectrometer and the method applied to the determination of arsenic species in river and tap water. The sample was loaded at $10 \mathrm{ml} \mathrm{min}^{-1}$ for $60 \mathrm{~s}$, followed by $0.2 \%$ tetrahydroborate at $5 \mathrm{ml} \mathrm{m^{-1 }}$ for $60 \mathrm{~s}$. The hydride was generated on the passage of $8 \mathrm{M} \mathrm{HCl}$ at $5 \mathrm{ml} \mathrm{min}^{-1}$ for $45 \mathrm{~s}$. The LOD for a $10 \mathrm{ml}$ sample volume was $0.004 \mu \mathrm{g} 1^{-1}$. This value may be compared (a) with the value of $0.04 \mu \mathrm{g} 1^{-1}$ obtained for previous FI-HGETAAS determination of As for which a sample volume of $0.500 \mathrm{ml}$ was used ${ }^{5}$ and (b) with the predicted value from the relevant FIDL equation for a $10 \mathrm{ml}$ sample of $0.011 \mu \mathrm{g} 1^{-1}$, showing that a significant decease in the contribution of the blank had been obtained by using the anion-exchange resin. A reference water sample (total As content $80 \mu \mathrm{g} \mathrm{l}^{-1}$ ) was accurately analysed and the interferences of nine cations and eight anions on $0.500 \mu \mathrm{g} 1^{-1}$ As were investigated.

\section{FI determination of lead}

Two approaches to the determination of lead have been under investigation. In the first, lead has been concentrated on a solid phase extractant and then eluted into a smaller volume of eluent for determination by FAAS. In the second approach, lead hydride has been generated, separated from solution and determined by AAS either with a quartz tube atomizer or, after in-atomizer trapping, with a graphite furnace atomizer. The possibility of in-atomizer trapping of lead in a slotted quartz tube in a flame has also been demonstrated.

\section{Analyte concentration by SPE}

Two manifold designs have been used for the concentration of lead as the diethyldithiocarbamate (DDC) complex by retention on a hydrophobic solid phase extractant material. In the first of these, ${ }^{15}$ the lead DDC complex was formed in the FI manifold by merging the sample stream with a solution of DDC buffered at $\mathrm{pH} 4-5$. The complex was retained on a small column of $\mathrm{C}_{18}$ silica and then eluted with acetonitrile. To avoid the loss of the DDC complex to the walls of the tubing, the confluence point was located inside the loop of the 'injection' valve so that in the 'elute' position all tubing in contact with the lead DDC complex was washed with eluent. This arrangement is shown in Fig. 3. The manifold was used in the determination of lead in water and fruit juice matrices at concentrations of 0.5 and $5 \mathrm{mg} \mathrm{l}^{-1}$. The enrichment factor, calculated as the ratio of calibration slopes, was about 50 and the detection limit was $6 \mu \mathrm{g} 1^{-1}$ for a sample volume of $5.8 \mathrm{ml}$ (2 min loading).

The second manifold was simpler. ${ }^{16}$ The lead-DDC complex was formed off-line at $\mathrm{pH} 9$ by the addition of the minimum amount of a DDC solution to the sample. The lead-DDC complex was retained on the GC stationary phase Chromosorb 102 (a styrene-divinylbenzene copolymer) and eluted into a small volume of ethanol delivered from an injection valve. This manifold is shown in Fig. 4. An enrichment factor of about 25 was obtained with an LOD of $2 \mu \mathrm{g}^{-1}$ for a sample volume of $4.4 \mathrm{ml}$ ( $2 \mathrm{~min}$ loading). The method was applied to the analysis of spiked tap water and artificial sea-water at concentrations around $0.1-0.4 \mathrm{mg}^{-1}$. A soil SRM (NIST SRM 2711 Montana Soil, lead content $1162 \mathrm{mg} \mathrm{kg}^{-1}$ ) was accurately analysed following digestion with nitric and hydrofluoric acid in a sealed vessel in a microwave field.

No problems either with sample loss to the walls of the tubing or with passage of sample matrix components to the nebulizer were encountered. This design of manifold would probably not be so suitable for use with ICP instrumentation which is less tolerant to the delivery of solutions of variable

(a)

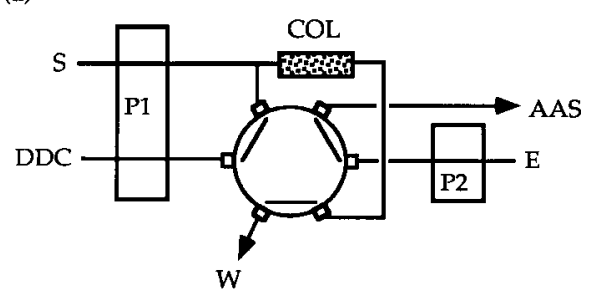

(b)

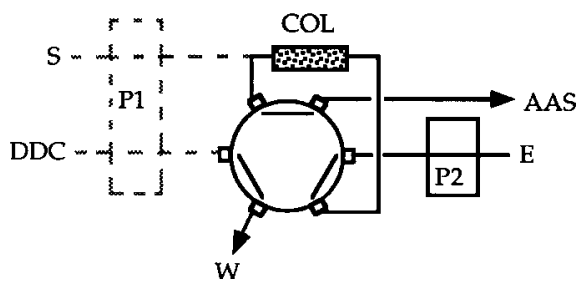

Fig. 3 Solid phase extraction manifold with confluence point in sample loop of six-port rotary valve. In the 'load' position (a), the sample, S, is merged with buffered DDC solution and the resulting complex is retained on the column, COL. In the 'inject' position (b), pump P1 is off while pump P2 keeps running, delivering eluent, E. The lead-DDC complex is back-flushed from the column to the spectrometer, FAAS, and any complex adhering to the walls of the tubing is also dissolved. $\mathrm{W}$ is waste. 

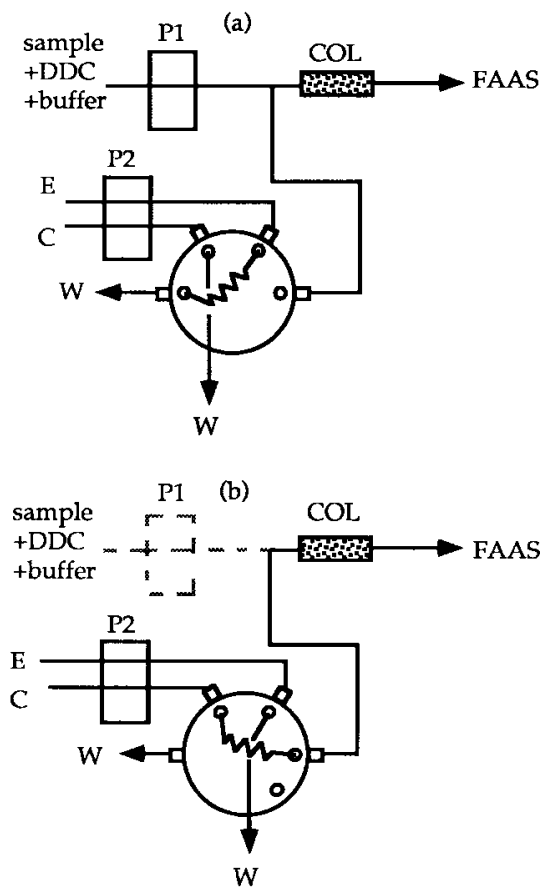

Fig. 4 Solid phase extraction manifold with Perkin-Elmer four-port rotary valve. In the 'load' position (a), the buffered sample and DDC are delivered to the column, COL. In the 'inject' position (b), pump $\mathrm{P} 1$ is off while pump P2 keeps running, delivering a discrete volume of eluent, E, via carrier C. The lead-DDC complex is flushed through the column to the spectrometer, FAAS. W is waste.

acidity and high dissolved solids. In these cases, a manifold design in which the matrix components were diverted to waste during loading would be more suitable.

\section{Hydride generation}

There has been relatively little interest in the HG determination of $\mathrm{Pb}$ in comparison with that in the $\mathrm{HG}$ determination of $\mathrm{As}$ and Se, for which HG-AAS is probably now the procedure of choice. Unlike the situation for the hydride-forming elements of Groups 14 and 15 of the Periodic Table, lead hydride is formed from a precursor in the highest oxidation state in solution, not the lowest. Even with an oxidative sample digestion or dissolution, lead is still in the +2 state and efficient generation of plumbane is only achieved in the presence of an oxidant. There are, therefore, a large number of possible reactions. Lead may participate in an oxidation reaction (from $\mathrm{Pb}^{\mathrm{II}}$ to $\mathrm{Pb}^{\mathrm{IV}}$ by the added oxidant), a hydride transfer reaction (the formation of plumbane from $\mathrm{Pb}^{\mathrm{IV}}$ ), and reduction reactions (tetrahydroborate is a strong reductant and also a hydride transfer reagent and can reduce $\mathrm{Pb}^{\mathrm{IV}}$ to $\mathrm{Pb}^{\mathrm{II}}$ and even to $\mathrm{Pb}^{0}$, which in turn may be oxidized by protons from the acid). In addition to the primary hydride generation reaction, tetrahydroborate reacts with acid (to form boric acid and hydrogen) and may react with the added oxidant in a redox reaction (most likely forming boric acid and hydrogen). It is perhaps surprising that any plumbane is generated at all, given the delicate balancing needed between the thermodynamics and kinetics of all of these possible reactions. In addition, some workers have found it advantageous to add a complexing agent (such as malate, tartrate or lactate), notionally to stabilize the $\mathrm{Pb}^{\mathrm{IV}}$ species. The conflicting literature regarding $\mathrm{Pb} \mathrm{HG}$ has recently been summarized. ${ }^{17,18}$

Various conditions for the generation of plumbane have been investigated, ${ }^{15,19}$ resulting in the conclusion that the greatest sensitivity for FI-HG-AAS with quartz tube or graphite furnace atomization is obtained in the presence of hexacyanoferrate(III) as 'oxidant' and hydrochloric acid. These findings are in agreement with those of several other workers. $^{20-23}$

A method was developed for the determination of lead in human urine. ${ }^{24}$ After optimization of the various flow injection parameters, the LOD was $0.08 \mu \mathrm{g} 1^{-1}$ for a sample volume of $0.500 \mathrm{ml}$. A quartz tube atomizer, a Nafion dryer (between the gas-liquid separator and the atomizer) and peak height quantification were used. An RM [NIST SRM 2670 Trace Metals in Urine with $\mathrm{Pb}$ concentrations of $10 \mu \mathrm{g}^{-1}$ (normal) (a suggested value) and $109 \mu \mathrm{g}{ }^{-1}$ (elevated) (a certified value)] was accurately analysed. ${ }^{25}$ However, when the possible interference of a chelating agent, such as EDTA (used in the treatment of patients with elevated lead), was investigated it was clear that the simple sample prepreatment of addition of hexacyanoferrate(III) and dilution with hydrochloric acid solution would not be suitable for the analysis of 'real' urine samples.

The method was modified by the addition of Sc, whose EDTA complex formation constant is $10^{5}$ times larger than that for PbEDTA, and the lead signal was restored. The modified procedure was applied blind to the analysis of 50 samples provided by the New York State Department of Health. Samples were diluted between 5- and 100-fold, and the solution injected contained $2.5 \times 10^{-4} \mathrm{M} \mathrm{Sc}, 5 \% \mathrm{~m} / \mathrm{v}$ hexacyanoferrate(III) and $0.1 \% \mathrm{HCl}$. Calibration with aqueous standards was possible. A comparison of the results of the determinations by FI-HG-AAS with those obtained by the New York State Department of Health (in whose laboratory an ETAAS procedure was used) is shown in Fig. 5, from which it can be seen that there is no significant difference at the $95 \%$ confidence level between these two sets of results.

The procedure has been further developed by trapping the hydride at $300{ }^{\circ} \mathrm{C}$ on the interior of a graphite furnace atomizer. ${ }^{25}$ The atomizer was first pretreated with $\operatorname{Ir}(120 \mu \mathrm{g})$. The LOD was $0.12 \mu \mathrm{g}^{-1}$ for a sample volume of $1.00 \mathrm{ml}$. However, after purification of the hexacyanoferrate(III) by passing a solution through a cation-exchange resin, the LOD was decreased to $0.03 \mu \mathrm{g}^{-1}$. Unfortunately, this procedure did not prove viable for routine use as the capacity of the resin to retain lead rapidly became exhausted. The procedure was used in a method for the determination of $\mathrm{Pb}$ in calcium supplements. Two materials (CVS brand) were examined: one contained $333 \mathrm{mg} \mathrm{Ca}+133 \mathrm{mg} \mathrm{Mg}+5 \mathrm{mg} \mathrm{Zn}$ per tablet and the other contained $500 \mathrm{mg} \mathrm{Ca}$ (from crushed oyster shells) per tablet. In terms of the lead content, the former contained $0.57 \mathrm{mg} \mathrm{kg}^{-1}$ (i.e., $0.43 \mu \mathrm{g}$ per tablet) and the latter $0.66 \mathrm{mg} \mathrm{kg}^{-1}$ (i.e., $0.43 \mu \mathrm{g}$ per tablet). The method was vali-

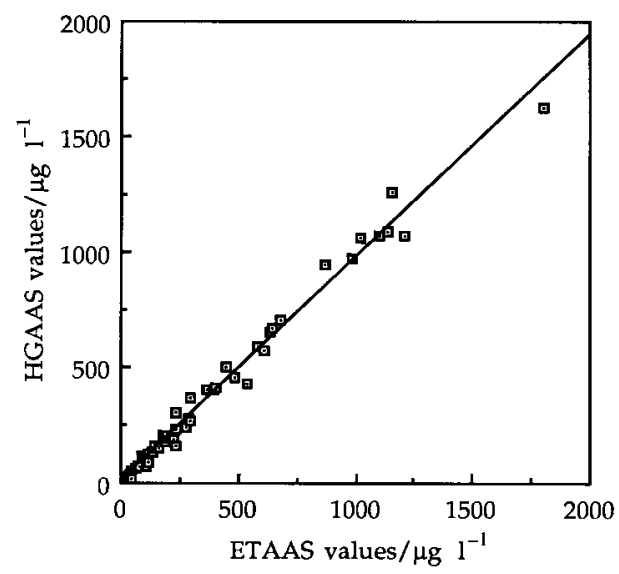

Fig. 5 Scatter plot of data for the determination of lead in urine by (a) HG-AAS (ordinate) and ETAAS (abscissa). The line is the unweighted least-squares linear regression fit to the data and has slope $0.963 \pm 0.041$, intercept $9.17 \pm 18.4$ and correlation coefficient 0.992 . The \pm terms are $95 \%$ confidence intervals. 
dated by the analysis of the materials by ETAAS methods (three different sample pre-treatment procedures were used) and by spike recoveries. Further studies of the $\mathrm{HG}$ of lead are in progress as the LOD for ETAAS is no better than that for quartz tube atomization, although the sensitivity of the former is much higher. It is concluded that the noise associated with the ETAAS procedure is much higher, and as the only significant difference between the two procedures is the in-atomizer trapping stage, it is thought that this process is, for some reason as yet unknown, less reproducible for plumbane than for other hydrides such as arsine and hydrogen selenide. Clearly improved LODs would be obtained with purer hexacyanoferrate(III) and work is in progress to find a routine method for the removal of the lead from this reagent.

Finally, in this saga of method development based on plumbane generation, a procedure based on trapping the hydride on the interior of a slotted quartz tube atomizer has been devised. ${ }^{26}$ The concept of trapping atom precursors on a cool surface in a flame was first described by Lau et al. ${ }^{27}$ and the concept of increasing atom residence time in the flame by the insertion of a slotted quartz tube was first described by Watling. ${ }^{28}$ The combination of the two procedures was proposed by Ataman and co-workers, ${ }^{29,30}$ who showed that it was possible to trap the precursor species in a fuel-lean flame and atomize in a fuel-rich flame produced either by rapidly changing the fuel flow or by the pulse nebulization of an organic solvent. They also showed that it was possible to dispense with the water-cooled tube and trap on the slotted tube. ${ }^{31}$ This concept has been extended ${ }^{26}$ by the introduction of the lead as the hydride into the slotted quartz tube in a fuel-lean flame. Essentially the same generation chemistry was used as for the previously developed HG methods. The lead was atomized on the injection of $50 \mu \mathrm{l}$ of isobutyl methyl ketone. The LOD was measured for three different sample volumes, 2.6, 5.2, and $7.8 \mathrm{ml}$, for which the LODs were 0.075 , 0.047 and $0.028 \mu \mathrm{g}^{-1}$, respectively. These data fit an FIDL plot of the equation $C_{\mathrm{L}}=0.175 / V+0.009$ with a correlation coefficient of 0.99 . The procedure was used as part of a method for the determination of $\mathrm{Pb}$ in two SRMs: NIST SRM 2709 San Joaquin Soil (19 $\left.\mathrm{mg} \mathrm{kg}^{-1}\right)$ and SRM 1515 Apple Leaves $\left(0.47 \mathrm{mg} \mathrm{kg}^{-1}\right)$ were analysed accurately.

Work is in progress ${ }^{32}$ on the determination of lead by a combination of SPE (the DDC complex on Chromosorb 102) with HG from the column eluent. The PbDDC was eluted with $0.3 \%$ ethanolic hydrochloric acid solution and merged with a hexacyanoferrate(III) stream prior to merging with tetrahydroborate. For quartz tube atomization, the LOD was $0.2 \mu \mathrm{g} 1^{-1}$ (i.e., about a factor of 10 improvement over the value obtained for elution with ethanol and determination by FAAS). The procedure was used as part of a method for the determination of $\mathrm{Pb}$ in wine over the concentration range 5 $-25 \mu \mathrm{g} 1^{-1}$. These results are interesting from two points of view: first, it appears that plumbane can be generated in the presence of DDC (the acid concentration may be high enough to release the lead from the complex) and ethanol, and second, it appears that DDC is capable of removing lead from the various complexing agents in the wine.

\section{Determination of cadmium}

The SPE chemistry, based on the retention of the DDC complex on $\mathrm{C}_{18}$ with elution by acetonitrile, has also been used for the determination of $\mathrm{Cd}$ in waters and fruit juices. ${ }^{15}$ Detection limits were measured for three different sample volumes, $0.79,2.36$ and $7.08 \mathrm{ml}$, for which values of $1.3,0.65$ and $0.39 \mu \mathrm{g}^{-1}$, respectively, were obtained. These data fit the FIDL equation $C_{\mathrm{L}}=0.799 / V+0.289$ with a correlation coefficient of 0.999 .

\section{Chemical vapour generation}

When tetrahydroborate is introduced into an acidified solution of cadmium, a volatile derivative is formed which can be either transported into a quartz tube atomizer in the light path of an atomic absorption spectrometer or trapped on the interior of a graphite furnace atomizer. The absorption signal produced from either atomizer is proportional to the cadmium concentration in the original solution and this phenomenon forms the basis of a viable method of analysis for cadmium. SanzMedel and co-workers ${ }^{33}$ made the interesting observation that, with the quartz tube atomizer, the procedure worked satisfactorily even when the tube was at room temperature. They provided convincing spectroscopic evidence ${ }^{34}$ that the absorbing species were indeed cadmium atoms and they proposed, therefore, that the procedure be called 'cold vapour' by analogy with the corresponding procedure for the determination of $\mathrm{Hg}$. It has been postulated that the species evolved from solution is cadmium hydride $\left(\mathrm{CdH}_{2}\right)$, but that this is sufficiently unstable that a significant proportion has decomposed to cadmium atoms during the time taken to pass from the gas-liquid separator to the atomizer. As cadmium atoms are not thermodynamically stable at room temperature with respect to the bulk metal, the cadmium atoms formed in this fashion are presumably kinetically 'stable'. These results have been confirmed by Guo and Guo ${ }^{35}$ and Kradtap. ${ }^{15}$

The best conditions for the generation of the precursor of the atoms are the subject of some debate. It has been reported that (a) a surfactant should be present, ${ }^{34}$ (b) that a considerable signal enhancement occurs in the presence of nickel, cobalt and thioure ${ }^{35}$ and (c) that potassium tetrahydroborate gives a significantly enhanced signal compared with that obtained in the presence of sodium tetrahydroborate. ${ }^{36}$ All of these claims have been investigated in some detail ${ }^{37}$ for a typical two-line flow injection system (i.e., the acidified sample is injected into an acid carrier which merges with an alkaline tetrahydroborate stream; after passage through a reaction coil, argon is merged and the mixture flows through a stripping coil to the gas-liquid separator). The findings are that a surfactant has no effect, nor has potassium tetrahydroborate. In the presence of $1 \%$ thiourea and $10 \mu \mathrm{g}^{-1} \mathrm{Ni}$ or $\mathrm{Co}$, the peak height signal is enhanced by $10-20 \%$ but a black precipitate rapidly forms in the presence of Co. In addition, it has been found that the surface of the atom cell plays no role in the atomization process. A Pyrex glass cell of smaller diameter than the 'standard' quartz cell can be used to increase the peak height signal. However, when a Nafion dryer was used to transport the volatile species to the atom cell, the signal was lost completely. A small open chamber, of volume about $5 \mathrm{ml}$, was used as a gas-liquid separator, which diverted only $8 \%$ of the cadmium to waste. It was observed that better signals were obtained if the acid concentration in the injected sample was lower than that in the carrier and that the length of tubing between the gas-liquid separator and the atom cell should be the minimum value possible. All of the evidence suggested that the signal characteristics were due to the kinetic features of an unstable analyte species. Interestingly, peak height was a more precise and robust quantitative parameter than peak area.

Procedures were developed for the accurate determination of Cd in NIST SRM 2711 Montana Soil at $41.7 \mathrm{mg} \mathrm{kg}^{-1}$ and NIST SRM 1515 Apple Leaves at $0.013 \mathrm{mg} \mathrm{kg}^{-1}$. The high lead content of Montana soil (1162 mg kg-1) interfered and was removed by coprecipitation with barium sulfate. In presence of thiourea and $\mathrm{Ni}$, the slopes of the standard additions calibration and that for aqueous standards were not significantly different. The LOD was $0.016 \mu \mathrm{g}^{-1}$ (only one value for a sample volume of $0.300 \mathrm{ml}$ has been obtained so far). 


\section{The eight-port flow injection valve}

While a variety of FI apparatus has been used in the experiments described above, the automation of several procedures has been greatly facilitated by the use of a twoposition 'eight-port' rotary valve. This designation is somewhat of a misnomer, as the device actually has 16 ports, eight on the stator and eight on the rotor. To illustrate the versatility of the device, three different uses are shown. First, the device has been used in a manifold for determinations of Se and As by HG-ETAAS in which the hydrides have been generated on the passage of acid through an column of anion-exchange resin on which the analyte and tetrahydroborate have been sequentially immobilized. The manifold and its operation are explained in Fig. 6. Second, a manifold for the separation of analyte from matrix by retention of the matrix on a solid phase extractant has been designed around this device. The manifold has been used for the separation of uranium (at concentrations up to $5000 \mathrm{mg} \mathrm{l}^{-1}$ ) from solutions of light elements ( $\mathrm{Al}, \mathrm{Be}, \mathrm{Li}$ and $\mathrm{Mg}$ ), allowing their determination at concentrations down to $1 \mu \mathrm{g} 1^{-1}$ by ICP-MS. ${ }^{38}$ The manifold and its operation are shown in Fig. 3 in ref. 38. Third, the device has been used in a manifold for the on-line microwaveassisted digestion of selenium compounds in urine, allowing the determination of Se in this matrix by HG. It has been shown $^{8}$ that it is possible to convert all Se compounds in human urine to selenite, by heating under reflux in the presence of bromine generated from the reaction of bromate with hydrobromic acid, but attempts to adapt this sample pretreatment to an on-line format had proved unsuccessful in terms of producing conditions which would convert trimethyl-

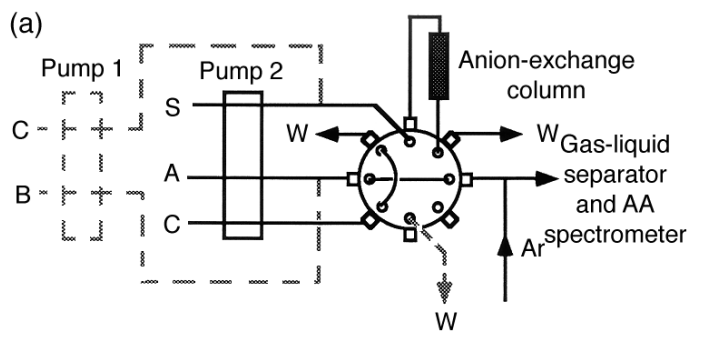

(b)
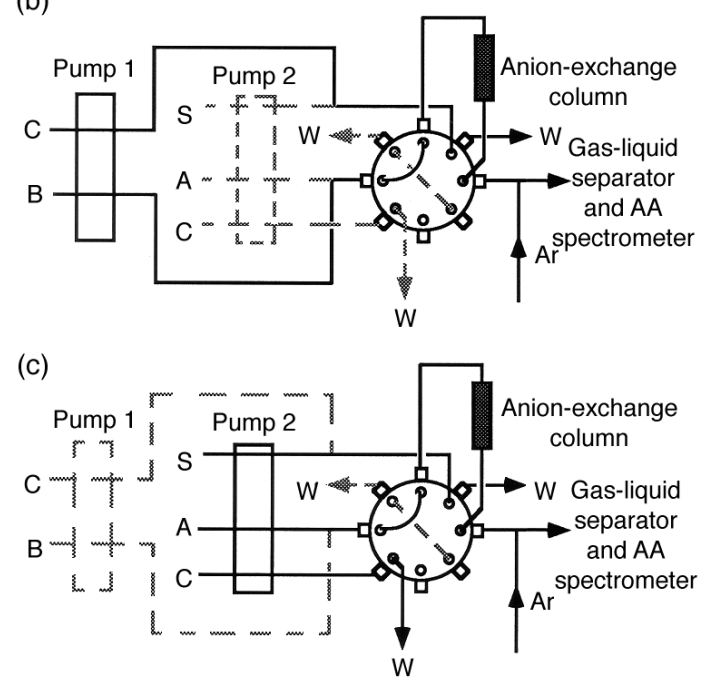

Fig. 6 Manifold for $\mathrm{HG}$ after preconcentration and immobilization of tetrahydroborate. With the pumps and valve as shown in (a), sample, $\mathrm{S}$, is loaded on to the column. The pumps and valves are then switched to the positions shown in (b), allowing tetrahydroborate, B, to be loaded on to the column. In the final stage, the pumps are switched as shown in (c) and the hydride generated on passage of the acid stream, A. Various intermediate stages, in which wash solution, $\mathrm{C}$, removes residual material, are not shown. $\mathrm{W}$ is waste.
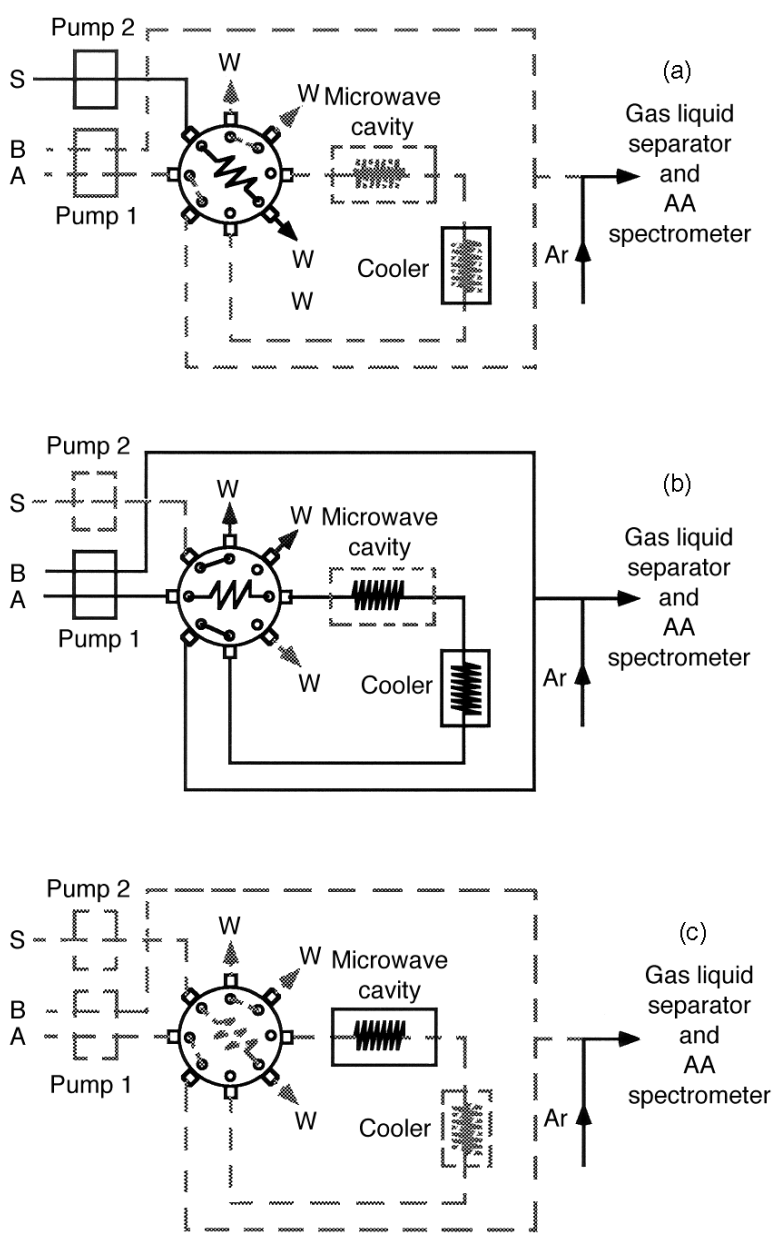

Fig. 7 Manifold for stopped-flow, closed-loop, microwave digestion. With the valve and pumps as shown in (a) the injection loop is loaded with sample solution. The pumps and valve are switched to the positions shown in (b) and the sample solution delivered by an acid carrier, A, into the digestion coil in the microwave cavity. When the valve is switched to the position shown in (c) and both pumps are off, the sample is digested in a sealed closed loop. The valve and pumps are then activated as shown in (b) and the digested sample pumped via the cooling coil to the $\mathrm{HG}$ part of the manifold and merged with a tetrahydroborate stream, B. Intermediate stages in which the probe is introduced into and removed from the graphite furnace are not shown.

selenonium (TMSe) (the Se species found in human urine most resistant to oxidative attack) into selenite. ${ }^{25}$ However, recently it has been found ${ }^{39}$ that a manifold design in which the sample zone is isolated in a closed loop in the microwave field produces conditions under which TMSe is converted into $\mathrm{Se}^{\mathrm{IV}}$. The manifold design is shown in Fig. 7. Under the same operating conditions, $\mathrm{Se}^{\mathrm{VI}}$, selenocystine, selenomethionine and selenoethionine were also quantitatively converted into $\mathrm{Se}^{\mathrm{IV}}$.

\section{Speciation of selenium and arsenic}

There is considerable research activity directed towards the development of methods for the separation and quantification of the various inorganic and organaometallic species of arsenic and selenium found in environmental and clinical samples. Numerous chromatographic (mainly HPLC) and nonchromaotgraphic procedures have been published, many of which incorporate AAS or ICP-MS detection. ${ }^{40,41}$ However, it appears that (a) is it difficult to achieve efficient $(>1000$ plates) LC separation of inorganic and methylated arsenic species in short times $(<5 \mathrm{~min})$ and (b) many clinical and 
biological materials contain numerous organoselenium compounds which are poorly resolved at short elution times or are unidentified, or both.

\section{Selenium speciation}

As part of a broad-based study of the biogeochemical transformations of selenium compounds and of the nature of selenium compounds in materials with anti-cancer properties, analytical methods are under development in which selenium compounds have been separated by HPLC and detected by ICP-MS. As the bulk of the studies so far have been concerned with plant and yeast materials, the targets have been amino acids and closely related species. Recent efforts have been devoted to improving the interface between the chromatograph and the spectrometer and to improving the performance of the chromatographic separation. Initial studies were made of ion-exchange separations and reversed-phase separations of amino acid derivatives. ${ }^{42,43}$ However, an ion-pair reversedphase procedure ${ }^{44,45}$ had superior performance in terms of (a) chromatographic separation, (b) ease of interfacing with the mass spectrometer and (c) the application of this procedure to the analysis of a variety of sample materials.

Further modifications of the conditions were made ${ }^{46}$ so that the composition of the mobile phase was $1 \% \mathrm{MeOH}+0.6 \%$ trifluoroacetic acid (TFA) $+25 \mathrm{mg} \mathrm{l}^{-1} \mathrm{Ge}$ (as internal standard) adjusted to $\mathrm{pH} 2$ with ammonia solution and the stationary phase was Waters SymmetryShield $\mathrm{RP}_{8}$. The improvement in chromatographic performance is shown in Fig. 8 for four selenium standard compounds. The injection volume was $10 \mu \mathrm{l}$ and the mobile phase flow rate was $1.0 \mathrm{ml} \mathrm{min}^{-1}$. Also contributing to the improved resolution were the use of a Meinhard nebulizer and a spray chamber of volume approximately $14 \mathrm{ml}$ containing a fixed $1 \mathrm{~cm}$ diameter glass impact bead.

Three different extraction procedures have been used. The first of these was a simple hot water extract as it is known ${ }^{47}$ that in the case of yeasts and allium vegetables, anti-cancer action is obtained with such extracts. The second was a water extract in the presence of an enzyme (protease XIV) ${ }^{48}$ which reduces polypeptides to dipeptides and the third extraction was with hydrochloric acid $(0.1 \mathrm{M})+$ ethanol $(95 \%)$. In each case the extraction was carried out for several hours, and the extract was filtered through a $0.45 \mu \mathrm{m}$ filter and a 10000 molecular mass cut-off filter (to remove polymeric material, including excess enzyme reagent). Total $\mathrm{Se}$ in the original material and in the residues after extraction and filtration was determined by a procedure in which the material was digested with nitric acid in a sealed vessel in a microwave field. This

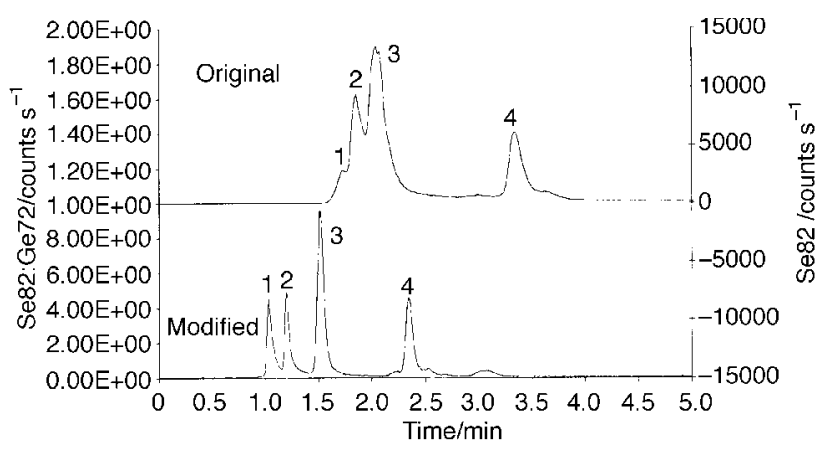

Fig. 8 Comparison between original and modified conditions for the separation of selenium compounds. The original conditions ${ }^{41}$ were a Zorbax SB-C $\mathrm{C}_{8}$ column with $2 \%$ methanol $+0.1 \%$ trifluoroacetic acid as mobile phase at a flow rate of $1.0 \mathrm{ml} \mathrm{min}{ }^{-1}$. The modified conditions (see text) were a Symmetryshield $\mathrm{RP}_{8}$ column with $1 \%$ methanol $+0.6 \%$ trifluoroacetic acid (adjusted to $\mathrm{pH} 2$ ). Peaks: 1, selenate; 2, selenite; 3 , selenocystine; and 4, Se-methyl-DL-selenocysteine. procedure was validated by the analysis of various reference materials. ${ }^{49}$ Although these studies are far from complete, some general observations have been made: for most materials studied a greater proportion of the total Se was removed by the enzymatic extraction than by the other procedures, suggesting that most selenium is incorporated into larger molecular structures that are broken down by the enzyme. The species profile depends on the material (not all yeasts give the same profile, for example) and the profile also depends on the concentration of the selenium. At this stage it difficult to draw any conclusions about the relative amounts of various selenium compounds which occur at natural concentrations. It has also been observed that for several different materials (including yeast, garlic, plankton, Brazil nuts and bacteria), the maximum amount of selenium that the organism can tolerate is about $2000 \mathrm{mg} \mathrm{kg}^{-1}$

A number of potential problems for the analytical methodology have also been identified: recovery of the selenium species from the column is a function of concentration, ranging from about $60 \%$ at $0.1 \mu \mathrm{g} 1^{-1}$ to over $90 \%$ at $100 \mu \mathrm{g} 1^{-1}$. Sensitivity is a function of species, with a $28 \%$ difference between the most sensitive and the least sensitive compounds and, in the determination of total Se, the sensitivity is a function of the acid concentration remaining after the digestion is complete. This problem has been noted by other workers and it may be overcome to a large extent by the addition of a mixture of water-soluble tertiary amines (known as CFA-C amines). ${ }^{50}$

Initial work on the identification of the selenium compounds has been based on retention time matching with standards. ${ }^{43-45}$ However, most of the compounds are still to be identified as no match with existing standards has been found. More recently, the approach of derivatizing with reagents designed to identify particular chemical functionalities has been attempted. These reagents have included hydrogen peroxide (as an oxidant), thiosulfate (as a reductant) and methanol or ethanol with sulfuric acid (as an esterifying agent). In this procedure, the chromatograms before and after reagent addition are compared to identify (a) which compounds may be oxidized or reduced (and whether the process is reversible) or (b) which compounds may be esterified. Future studies ${ }^{51}$ will involve the use of LC-MS (electrospray ionization with an ion trap spectrometer) for which initial studies on known selenium compounds indicate that the spectral interpretation may not be so complicated (as the presence of Se in a fragment ion is easily deduced from the characteristic isotope pattern), but that the sensitivity of this form of LC-MS is comparatively low.

\section{Arsenic speciation}

A common preservative for structural timber is chromated copper arsenate. The timber is treated under pressure with an aqueous solution of the oxides. As part of an on-going study into the fate of arsenic leached from pressure-treated timber into soils, a method for the determination of $\mathrm{As}^{\mathrm{III}}, \mathrm{As}^{\mathrm{V}}$ and the two methylated forms of arsenate (discussed earlier) in soils has been under development. The hypothesis to be examined is that the inorganic arsenic which gets into the soil is transformed to volatile methylated species by the action of soil micro-organisms. Initially, an anion-exchange HPLC separation was devised ${ }^{52}$ and it was shown, on the basis of ultrasound-assisted extraction with methanol+hydrochloric acid $(50 \%+10 \%)$, that an arsenic-containing reference material, NIST SRM 2704 Buffalo River Sediment, contained arsenite and dimethyl arsinate. Following the success of the ion-pair procedure for the separation of selenium amino acids, work has been directed ${ }^{53}$ to the development of a similar procedure for the separation of these four arsenic species. A 


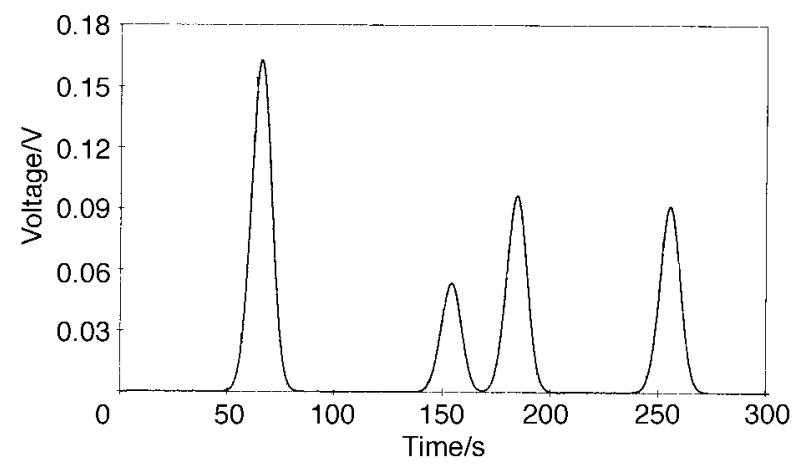

Fig. 9 Elution profile for arsenic species separated by reversed phase ion-pair HPLC with HG-AAS detection. The elution order is arsenite, dimethylarsonate, monomethylarsinate and arsenate. The flow rate was $1.0 \mathrm{ml} \mathrm{min}^{-1}$.

procedure had been devised based on the use of a Waters Resolve $\mathrm{C}_{18}$ column with $6 \mathrm{~mm}$ potassium dihydrogenphosphate $+2 \mathrm{~mm}$ tetrabutylammonium hydroxide $+0.2 \%$ $\mathrm{MeOH}$ at pH 5.7 (adjusted with phosphoric acid) as the mobile phase. For detection by post-column HG-AAS with quartz tube atomization, LODs between 0.5 and $2 \mu \mathrm{g} 1^{-1}$ have been obtained for a $100 \mu$ injection volume. The chromatographic profile is shown in Fig. 9.

The extraction of these arsenic species from soil is currently being studied. ${ }^{53}$ Obtaining quantitative recovery without transformation is proving to be difficult. A variety of extractants have been used, including tetrabutylammonium hydroxide and phosphoric acid (at a variety of $\mathrm{pH}$ values with potassium dihydrogenphosphate as buffering agent). The possible roles of ultrasound and microwave energies are also being studied. So far it may be concluded that it is possible to extract MMA and DMA, but $\mathrm{As}^{\mathrm{III}}$ is strongly bound immediately on spiking into a soil matrix and, so far, it has not proved possible to recover all of this species. The application of microwave energy results in the conversion of some $\mathrm{As}^{\mathrm{III}}$ into $\mathrm{As}^{\mathrm{V}}$ and therefore some of the earlier good recoveries for $\mathrm{As}^{\mathrm{V}}$ are called into question. This is a difficult analytical problem and there is still a long way to go with this method development.

\section{Conclusions}

Flow-based procedures, in which sample pre-treatment chemistry is performed in narrow-bore conduits, have a number of characteristics which can lead to the development of improved analytical methods. In addition to providing a closed, contamination-free environment for the handling of samples, flow procedures allow for the automated or semiautomated implementation of analyte and matrix separations that would be tedious, time consuming and prone to contamination if performed in a batch mode. In addition, many flow procedures allow for simple direct coupling between the sample pre-treatment stages and the instrumental measurement stage of the overall method. Flow procedures are a good way to implement solid phase extraction and chemical vapour generation procedures, although the usual increase in reagent consumption as the sample volume increases produces a rectangular hyperbolic relationship (the so-called FIDL equation) between sample volume and LOD which is not asymptotic to the concentration axis. There are, therefore, limits to the extent to which LODs can be improved by increasing the sample volume. However, in the case of chemical vapour generation, the use of an immobilized reagent removed the dependence of reagent blank on sample volume and a way has been opened up for further improvements in detection limits. The dependence of LOD on sample volume is a useful performance characteristic and the relevant information should be included in publications.

There are distinct possibilities for the use of $\mathrm{HG}$ in methods for the determination of $\mathrm{Pb}$ and $\mathrm{Cd}$, and such procedures could become as popular as those for the determination of As and Se. The generation of lead hydride is greatly facilitated by the presence of hexacyanoferrate(III). Cadmium may be determined by AAS at room temperature.

For the determination of various selenium and arsenic species in environmental and food samples, separation by reversed-phase ion-pair HPLC with element-specific detection seems to be a viable stage in the overall method, despite some of the difficulties encountered related to recovery from the column and the dependence of sensitivity on chemical factors. In the case of the selenium compounds, the identification of species responsible for large numbers of peaks in the chromatograms remains a major area of further work. For both As and Se compounds, the problems of extraction of the compounds from solid matrices also remain as a major area for further work.

Many helpful discussions with the following collaborators and co-workers are gratefully acknowledged: Peter Uden (University of Massachusetts, Amherst, MA), Eric Block (SUNY, Albany, NY), Helen Crews (MAFF, UK), Erik Larsen (Danish Veterinary and Food Administration), Latif Elci (Erciyes University, Turkey), Nusret Ertas, (Middle East Technical University, Ankara, Turkey), Patrick Parsons, (New York State Department of Health), Walter Slavin (Bonaire Technologies), Pablo Carrero (University of the Andes, Merida, Venezuela) and Susan McIntosh, Chris Hanna, Frank Fernandez, Glen Carnrick, Gerhard Schlemmer and Eric Denoyer (Perkin-Elmer). In addition, the efforts of the following graduate students, who conducted much of the experimental work described above, is gratefully acknowledged: Zikri Arslan, Susan Bird, Robert Ellis, Hakan Gurleyuk, Mihaly Kotrebai, Supaporn Kradtap, Nils Sundin, Cesar Vargas and Peter Yehl.

\section{References}

1 R. E. Sturgeon, J. Anal. At. Spectrom., 1998, 13, 351

2 J. F. Tyson, Microchem. J., 1992, 45, 143.

3 S. J. Hill, J. B. Dawson, W. J. Price, I. L. Shuttler and J. F. Tyson, J. Anal. At. Spectrom., 1997, 12, 327R.

4 J. Dedina and D. Tsalev, Hydride Generation Atomic Absorption Spectrometry, Wiley, Chichester, 1995.

5 J. F. Tyson, R. I. Ellis, S. A. McIntosh and C. P. Hanna, J. Anal. At. Spectrom., 1998, 13, 17.

6 H. Goenaga Infante, M. L. Fernandez-Sanchez and A. SanzMedel, J. Anal. At. Spectrom., 1996, 11, 571.

7 Analytical Methods Committee, Analyst, 1987, 112, 199.

8 J. F. Tyson, N. G. Sundin, C. P. Hanna and S. McIntosh, Spectrochim. Acta, Part B, 1997, 52, 1773.

9 S. Tesfalidet and K. Irgum, Anal. Chem., 1989, 61, 2079.

10 S. Tesfalidet and K. Irgum, Fresenius' J. Anal. Chem., 1991, 341, 532.

11 P. E. Carrero and J. F. Tyson, Analyst, 1997, 122, 915.

12 P. E. Carrero and J. F. Tyson, Spectrochim. Acta, Part B, in the press.

13 P. E. Carrero and J. F. Tyson, Spectrochim. Acta, Part B, submitted for publication.

14 P. E. Carrero and J. F. Tyson, J. Anal. At. Spectrom., submitted for publication.

15 S. Kradtap, MS Thesis, University of Massachusetts, Amherst, MA, 1996.

16 L. Elci and J. F. Tyson, work in progress.

17 J. Dedina and D. L. Tsalev, Hydride Generation Atomic Absorption Spectrometry, Wiley, Chichester, 1995, p. 288.

18 C. Camara and Y. Madrid, Analyst, 1994, 119, 1647.

19 R. E. Ellis, L. Elci, N. Ertas, C. Vargas and J. F. Tyson, work in progress.

20 R. Thao and H. Zhou, Fenxi Huaxue, 1985, 13, 283.

21 P. Zhang and Z. Hu, Fenxi Huaxue, 1987, 15, 404. 
22 H. Chen, J. Wu and I. D. Brindle, Talanta, 1994, 42, 353.

H. O. Huag, Spectrochim. Acta, Part B, 1996, 51, 1425.

24 J. F. Tyson, R. I. Ellis, N. Ertas, F. Fernandez and S. A. McIntosh, Clin. Chem., submitted for publication.

25 R. I. Ellis, PhD Dissertation, University of Massachusetts, Amherst, MA, 1997.

26 N. Ertas, Z. Arslan and J. F. Tyson, work in progress.

27 C. M. Lau, A. Held and R. Stephens, Can. J. Spectrosc., 1976, 21, 100.

28 R. J. Watling, Anal. Chim. Acta, 1977, 94, 181.

29 O. Y. Ataman, N. Ertas, R. S. Helles and S. Kumser, Paper presented at the FACSS XX Annual Conference, Detroit, MI, October 17-22, 1993, Paper 267.

30 N. Ertas, S. Kumser, D. Karadeniz, R. S. Helles and O. Y. Ataman, Paper presented at the FACSS XXIII Annual Conference, Kansas City, MO, September 29-October 4, 1996, Paper 607.

31 N. Ertas, D. Karadeniz and O. Y. Ataman, Paper presented at the XXX Colloquium Spectroscopicum Internationale, Melbourne, September 21-26, 1997, Paper C66.

32 L. Elci and J. F. Tyson, work in progress

33 A. Sanz-Medel, M. R. Fernandez de la Campa, M. C. ValdezHevia and M. Bordel, Anal. Proc., 1995, 32, 49.

34 A. Sanz-Medel, M. R. Fernandez de la Campa, M. C. ValdezHevia and M. Bordel, Anal. Chem., 1995, 67, 2216.

35 X.-W. Guo and X.-M. Guo, Anal. Chim. Acta, 1995, 310, 377.

36 H. Matusiewicz, M. Kopras and R. E. Sturgeon, Analyst, 1997, $122,331$.

37 C. Vargas-Razo and J. F. Tyson, J. Anal. At. Spectrom., submitted for publication.
38 P. E. Becotte-Haigh, J. F. Tyson and E. R. Denoyer, J. Anal. At. Spectrom., 1998, 13, 1327

39 P. E. Carrero and J. F. Tyson, work in progress.

40 S. J. Hill, J. B. Dawson, W. J. Price, I. L. Shuttler, C. M. M. Smith and J. F. Tyson, J. Anal. At. Spectrom., 1998, 13, $131 \mathrm{R}$.

41 J. R. Bacon, J. S. Crain, L. Van Vaeck and J. G. Williams, J. Anal. At. Spectrom., 1998, 13, 171R.

42 H. Ge, PhD Dissertation, University of Massachusetts, Amherst, MA, 1997.

43 S. M. Bird, H. Ge, P. C. Uden, J. F. Tyson, E. Block and E. Denoyer, J. Chromatogr. A, 1997, 789, 349.

44 S. M. Bird, P. C. Uden, J. F. Tyson, E. Block and E. Denoyer, J. Anal. At. Spectrom., 1997, 12, 785.

45 S. M. Bird, M. Kotrebai, P. C. Uden, J. F. Tyson, E. Block and E. Denoyer, Fresenius' J. Anal. Chem., 1998, 362, 447.

46 M. Kotrebai, P. C. Uden and J. F. Tyson, work in progress.

47 C. Ip and D. J. Lisk, Carcinogenesis, 1995, 16, 2649.

48 N. Gilon, M. Potin-Gauthier and M. Astruc, J. Chromatogr. A, 1996, 750, 327.

49 S. M. Bird, PhD Dissertation, University of Massachusetts, Amherst, MA, 1998.

50 A. Krushevska, M. Kotrebai, A. Lasztity, R. M. Barnes and D. Amarsiriwardena, Fresenius' J. Anal. Chem., 1996, 355, 793.

51 I. Kaltashov, M. Kotrebai, P. C. Uden and J. F. Tyson, work in progress.

52 P. M. Yehl and J. F. Tyson, Anal. Commun., 1997, 34, 49.

53 H. Gurleyuk, P. C. Uden and J. F. Tyson, work in progress.

Paper 8/07025C 